\title{
INTRA-BEAM SCATTERING SCALING FOR VERY LARGE HADRON COLLIDERS*
}

\author{
J. Wei ${ }^{\dagger}$ and G. Parzen \\ Brookhaven National Laboratory, New York, USA
}

\begin{abstract}
For Very Large Hadron Colliders (VLHC), flat hadron beams [2] with their vertical emittance much smaller than their horizontal emittance are proposed to maximize the design luminosity. Emittance growth caused by intra-beam scattering (IBS) is a concern on the realization of such flat-beam conditions. Based on existing IBS formalism on beams of Gaussian distribution, we analytically derive [6] the IBS growth rate and determine the IBS limit on the aspect ratio for a flat beam.
\end{abstract}

\section{INTRODUCTION}

Intra-beam scattering (IBS) plays an important role in the design and performance of hadron colliders [1]. Emittance growth and beam loss caused by intra-beam scattering degrades machine luminosity. The dynamic exchange and growth of beam temperature further complicates beam behavior at storage. Similar problem exists for proposed Very Large Hadron Colliders (VLHC).

Recently, flat hadron beams with their vertical emittance much smaller than their horizontal emittance have been proposed to maximize the VLHC design luminosity [2]. For such beams, intra-beam scattering potentially limits the achievable aspect ratio between the vertical and horizontal emittance. This paper attempts to describe the principle scaling behavior of the growth rates and, based upon which, the limiting aspect ratio of a flat beam determined by intra-beam scattering.

Intra-beam scattering theory often assumes a Gaussian beam distribution $[3,4,5,6]$ in the six-dimensional phase space, which is usually a good approximation if the beam is in a strong diffusive process caused by e.g. intra-beam scattering [7] and synchrotron radiation. Based on existing IBS formalism on beams of Gaussian distribution, we re-present in Section 2 analytical forms of the growth rates assuming a machine lattice consisting of regular cells. Dependence of the growth rates on the beam charge state, mass, energy, phase-space area, and the machine transition energy is obtained. Based on these formulae, we derive in Section 3 the scaling laws for the beam in the VLHC regime, and discuss the IBS limit on the flat beam aspect ratio. The approximation of the Coulomb logarithm is discussed in Section 4. A numerical example is given in Section 5. Further discussions are given in Section 6.

\footnotetext{
* Work performed under the auspices of the US Department of Energy
}

$\dagger$ wei1@bnl.gov, on joint appointment at ORNL and BNL

\section{ANALYTICAL FORMULAE}

The growth of particle beam under intra-beam scattering is usually described by the relative time derivatives of rms horizontal betatron amplitude $\sigma_{x}$, vertical amplitude $\sigma_{y}$, and fractional momentum deviation $\sigma_{p}$, respectively. Assume that the scatterings mostly occur at small scattering angles, and that the particle distribution remains Gaussian in the six-dimensional phase space. For an accelerator consisting mostly of regular cells where the variation in quantity $D_{p} / \beta_{x}^{1 / 2}$ ( $D_{p}$ is the dispersion, $\beta_{x}$ is the horizontal amplitude function.) is small along the circumference, the growth rates are obtained approximately for a beam with energy much higher than the transition energy of the machine $\left(\gamma \gg \gamma_{T}\right)$ as [5]

$$
\left[\begin{array}{c}
\frac{1}{\sigma_{p}} \frac{d \sigma_{p}}{d t} \\
\frac{1}{\sigma_{x}} \frac{d \sigma_{x}}{d t} \\
\frac{1}{\sigma_{y}} \frac{d \sigma_{y}}{d t}
\end{array}\right]=\frac{\pi N r_{0}^{2} m_{0} c^{2} L_{c}}{32 \gamma \epsilon_{x} \epsilon_{y} S}\left[\begin{array}{l}
\left(1-d^{2}\right)\left(\frac{1}{a}+\frac{1}{b}\right) \\
d^{2}\left(\frac{1}{a}+\frac{1}{b}\right) \\
-b^{2}\left(\frac{2}{b}-\frac{1}{a}\right)
\end{array}\right]
$$

where the particle motions in the horizontal and vertical directions are assumed to be decoupled, $r_{0}=Z^{2} e^{2} / A m_{0} c^{2}$ is the particle classical radius, $Z$ is the charge number, $A$ is the atomic mass number, $m_{0}$ is the unit atomic mass, $\gamma$ is the Lorentz factor, $\sigma_{s}$ is the rms bunch length, $N$ is the number of particles per bunch, and

$$
d=\frac{D_{p} \sigma_{p}}{\left(\sigma_{x}^{2}+D_{p}^{2} \sigma_{p}^{2}\right)^{1 / 2}}, \quad a=\frac{\beta_{x} d}{D_{p} \gamma}, \quad b=\frac{\beta_{y} \sigma_{x}}{\beta_{x} \sigma_{y}} a .
$$

The quantity $d<1$ is the effective ratio between the longitudinal and horizontal amplitude. The Coulomb logarithm factor $L_{c}$ has a value around $20[8,5]$, not sensitive to detailed beam configuration. The normalized rms transverse emittance is $\epsilon_{x, y}=\beta \gamma \sigma_{x, y}^{2} / \beta_{x, y}$, and the rms longitudinal bunch area (per nucleon) in phase space is $S=\pi m_{0} c^{2} \gamma \beta \sigma_{s} \sigma_{p} / c A$.

The growth rates are linearly proportional to the number of particle $N$ in the beam bunch, and are strongly dependent $\left(\sim Z^{4} / A^{2}\right)$ on the charge state of the particle. Except for the form factors $d, a$, and $b$ that depend on the ratio of the beam amplitudes in different directions, the rates are inversely proportional to the six-dimensional phase space area.

In the so-called negative-mass regime when the beam energy is much higher than the machine transition energy, the 
growth in horizontal direction is mostly produced from the variation of the betatron orbit during the exchange of particle momenta $\left(a^{2} \ll d^{2}\right)$ [9]. The growths in horizontal and longitudinal amplitudes are therefore proportional to each other. Asymptotically, their rms beam sizes are related by the average dispersion by [5]

$$
D_{p} \sigma_{p} \approx \sigma_{x}
$$

Thus, $d^{2} \approx 1 / 2$. On the other hand, the vertical beam size can either grow or damp depending on the ratio of transverse beam sizes.

\section{FLAT BEAM REGIME}

We define flat-beam aspect ratio

$$
\kappa \equiv \epsilon_{y} / \epsilon_{x}<1
$$

and assume that the average horizontal and vertical lattice amplitude functions are the same,

$$
\left\langle\beta_{x}\right\rangle \approx\left\langle\beta_{y}\right\rangle
$$

Thus

$$
b \approx \kappa^{-1 / 2} a>a
$$

The vertical beam size will grow if $2 \kappa^{1 / 2}<1$. The condition for a vertical damping is given by

$$
\kappa \geq \frac{1}{4}
$$

Since $\gamma \gg \gamma_{T}$, the vertical growth rate is usually small even though Eq. 7 is not satisfied. In order for the vertical growth rate to be of the same order as the horizontal growth rate, the beam has to be extremely flat, or

$$
\kappa^{1 / 2} \approx \frac{\gamma_{T}}{\gamma} \ll 1
$$

The growth rates can be explicitly written in terms of $\kappa$ as

$$
\left[\begin{array}{c}
\frac{1}{\sigma_{p}} \frac{d \sigma_{p}}{d t} \\
\frac{1}{\sigma_{x}} \frac{d \sigma_{x}}{d t} \\
\frac{1}{\sigma_{y}} \frac{d \sigma_{y}}{d t}
\end{array}\right]=\frac{\pi N r_{0}^{2} m_{0} c^{2} L_{c}}{32 \kappa \gamma_{T} \epsilon_{x}^{2} S}\left[\begin{array}{l}
\left(1-d^{2}\right) / d \\
d \\
-\left(\frac{\gamma_{T}}{\gamma}\right)^{2} \frac{d}{\kappa}\left(2 \kappa^{1 / 2}-1\right)
\end{array}\right]
$$

The dependence of horizontal and longitudinal growth on the energy of the beam, which appears only in the form factor $d$, is usually weak.

\section{COULOMB LOGARITHM}

Derivation of the analytical form of the growth rates is simplified by the assumption that the Coulomb logarithm is not sensitive $[8,5]$ to detailed beam parameter variations.
Table 1: Summary of VLHC parameter example.

\begin{tabular}{lrr}
\hline Energy, $E_{s}$ & 50 & $\mathrm{TeV}$ \\
Lorentz factor, $\gamma$ & 5.33 & $10^{4}$ \\
Circumference & 95 & $\mathrm{~km}$ \\
Transition energy, $\gamma_{T}$ & 53 & \\
Average $\beta_{x, y}$ in arc & 500 & $\mathrm{~m}$ \\
Average dispersion in arc, $D_{p}$ & 9 & $\mathrm{~m}$ \\
Initial bunch intensity & 1.25 & $10^{10}$ \\
Initial rms emittance, $\pi \epsilon_{x}$ & 2 & $\pi \mathrm{mm} \cdot \mathrm{mr}$ \\
Typical rms bunch area, $S$ & 0.3 & $\mathrm{eV} \cdot \mathrm{s}$ \\
Typical rms spread in $\Delta p / p$ & 2 & $10^{-5}$ \\
Typical rms bunch length & 0.02 & $\mathrm{~m}$ \\
\hline
\end{tabular}

The singularity in the integral [3] is avoided by introducing a finite minimum scattering angle, which corresponds to a maximum impact parameter of the order of the beam radius. In the case of a vertically flat beam, this maximum impact parameter $\bar{b} \approx 2 \sigma_{x}$ is approximately twice the rms horizontal beam size. Using typical VLHC parameters listed in the next section, it is shown that the Coulomb logarithm factor can still be approximated as

$$
L_{c} \approx \ln \left[\frac{4 \beta^{2} \bar{b} \sigma_{p}^{2}\left(1-d^{2}\right)}{r_{0}\left(a^{2}+b^{2}\right)}\right] \approx \ln \left[\frac{4\left\langle\sigma_{x}\right\rangle \sigma_{p}^{2} \gamma^{2}}{r_{0} \gamma_{T}^{2}\left(1+\kappa^{-1}\right)}\right]
$$

With parameters listed in Table $1, L_{c}$ is about 26 when $\kappa$ is 1 , and is about 22 when $\kappa$ is 0.01 .

\section{EXAMPLE}

As an example, we consider a VLHC case [10] as shown in Table 1. With these parameters, we have $\left\langle\sigma_{x}\right\rangle \approx 1.4 \times$ $10^{-4} \mathrm{~m}, D_{p} \sigma_{p} \approx 1.8 \times 10^{-4} \mathrm{~m}$, and $d^{2} \approx 0.62$. Although the vertical beam size starts to grow under intra-beam scattering when

$$
\kappa<\frac{1}{4}
$$

the vertical growth becomes significant only when

$$
\kappa<\left(\frac{\gamma_{T}}{\gamma}\right)^{2} \approx 10^{-8}
$$

which is much more relaxing than those from other coupling mechanisms.

To give an order-of-magnitude estimate on the IBS growth rates, we assume that $\kappa=0.01$. The growth rates are given by

$\left[\begin{array}{c}\frac{1}{\sigma_{p}} \frac{d \sigma_{p}}{d t} \\ \frac{1}{\sigma_{x}} \frac{d \sigma_{x}}{d t} \\ \frac{1}{\sigma_{y}} \frac{d \sigma_{y}}{d t}\end{array}\right]=9.3 \times 10^{-5}\left[\begin{array}{l}0.48 \\ 0.79 \\ 6.2 \times 10^{-5}\end{array}\right]\left[\mathrm{s}^{-1}\right]$ 
The growth time in the horizontal and longitudinal directions is of the order of a few hours, while the growth in the vertical direction is negligible.

\section{DISCUSSIONS}

Using analytical expressions, we have obtained the scaling laws for the beam size growth rates under intra-beam scattering in the VLHC regime. From the point of view of intra-beam scattering, the vertical emittance growth becomes significant only when the condition of Eq. 8 is satisfied. With the present VLHC parameters, intra-beam scattering does not impose a practical limitation on the preservation of a flat beam. The IBS growth rates for the horizontal and longitudinal beam amplitudes are of the same order as the radiation damping rates.

The expressions presented in this paper is a simplification of the formalism pioneered by Piwinski [3] and developed by Martini [4], Parzen [5] and others. Detailed calculation can be performed using their full integral expressions taking into account general lattice information. It has been assumed that the beam distribution remains Gaussian in the phase space, and that beam loss due to machine acceptance limitation is negligible. In the case that these assumptions are not valid, Fokker-Planck approaches can be used to study general beam evolution under intra-beam scattering [7].

The intra-beam scattering results obtained in this paper also apply to electron storage rings where the beam energy is typically much higher than the transition energy of the machine.

\section{ACKNOWLEDGEMENTS}

One of the authors (JW) would like to thank Dr. V. Lebedev and Dr. A. Burov for many useful discussions.

\section{REFERENCES}

[1] J. Wei and M. Harrison, The RHIC Project - Design, Status, Challenges, and Perspectives, Proc. XVI RCNP Osaka International Symposium on Multi-GeV High-Performance Accelerators and Related Technology, Osaka, Japan (March 1997).

[2] S. Peggs, M. Harrison, F. Pilat, M. Syphers, Flat Beams in a $50 \mathrm{TeV}$ Hadron Collider, Proc. 1997 Particle Accelerator Conference, Vancouver, p. 95 (1997).

[3] A. Piwinski, Intra-Beam-Scattering, CERN 92-01, Proc. CERN Accelerator School, Gifsur-Yvette, Paris, 1984, p405.

[4] M. Martini, Intrabeam Scattering in the Acol-AA Machines, CERN PS/84-9 (AA) (1984).

[5] G. Parzen, Intrabeam Scattering at High Energies, Nucl. Instr. Meth. A256, 231 (1987); G. Parzen, Proc. 1988 European Particle Accelerator Conference, Rome, p.821.

[6] J. Wei, Evolution of Hadron Beams under Intrabeam Scattering, Proc. 1993 Particle Accelerator Conference, Washington, D.C. (May 1993) p.3653.
[7] J. Wei and A.G. Ruggiero, Beam Life-Time with Intrabeam Scattering and Stochastic Cooling, Proc. 1991 Particle Accelerator Conference, San Francisco, p.1869.

[8] J. Bjorken and S. Mtingwa, Intrabeam Scattering, Particle Accelerators 13, 115 (1983).

[9] J. Wei, Intensity Dependent Effects in RHIC, Proc. Workshop on Instabilities of High Intensity Hadron Beams in Rings, AIP 496, ed. T. Roser and S.Y. Zhang, (1999) p. 197.

[10] This list of parameters is used during the Mini-Workshop on the Effects of Synchrotron Radiation in VLHC, Brookhaven National Laboratory, September 2000 (web site http://www.vlhc.org). 